\title{
Modeling of Lithium-ion Battery for Charging/Discharging Characteristics Based on Circuit Model
}

\author{
https://doi.org/10.3991/ijoe.v13i06.6799 \\ Zhai Haizhou \\ Heze University, Heze, China \\ kateng $0204 @ 126$.com
}

\begin{abstract}
The paper describes the principle and charging/discharging characteristics of lithium-ion battery. It is as the research objects that Lithium-ion batteries with positive and negative materials of LiyMn2O4 and LixC6 were selected. A mathematical model is made for simulating the electrochemical behavior of Lithium-ion batteries. It is established that the electrochemical cell model and the one RC cell model based on aforementioned. The case of voltage, current, temperature, SOC and the charging/discharging characteristics were studied. The model is dynamic, and it reflects the transient state of the battery output. This is part of a laboratory setup used to test power system of an electric vehicle or hybrid vehicle to simulate electrochemical energy storage. The results show that accurate battery charging/discharging strategy management and SOC measurement can be achieved.
\end{abstract}

Keywords-lithium-ion battery, charging/discharging characteristics, SOC

\section{$1 \quad$ Introduction}

In recent years, environmental problems caused by fuel vehicles and fuel economy become more and more serious. The vehicles of new energy, which is green, environmentally friendly and economical, is an important goal for economic and social development of many countries, but also the future development direction of the vehicle. Electric vehicle (EV) is a vehicle with zero pollution emissions, mileage and fuel cars can be mutually comparable electric vehicles. Battery mileage comparable to that of fuel-powered vehicles. Cars (electric cars, EV) is a battery as a power source of a zero pollution emissions, mileage and fuel cars can be mutually comparable electric vehicles. It is reported that load capacity statistics data of new projects in on energy storage technology, lithium-ion battery system is the highest capacity of $113.8 \mathrm{MW}$, accounting for $54.7 \%$ of the global capacity total in 2015 as stated in [1-3]. In addition, lithium-ion battery has become the mainstream of automotive power battery. Lithium-ion batteries have many advantages, such as high energy density, high opencircuit voltage, small self-discharge, no memory effect, and so on. Lithium-ion batteries have good prospects for development in the field of electric vehicles. 
As the driving force of electric vehicles, the battery technology determines how healthy and rapidly electric vehicles developing. Therefore, the design and performance optimization of lithium-ion batteries has become an important research direction. Lithium-ion batteries as a typical electrochemical energy storage system, which including electrochemical reaction, transfer of mass, charge and heat processes as stated in $[4,5]$. The research of lithium-ion battery is rich-contented, and complex. At present, modern means of electrochemical analysis and instruments, making the traditional experimental research methods to a certain extent, can clarify the battery material on the battery performance mechanism, the nature of the characteristics of the material properties, and then evaluate the battery performance, but there is limitations of single variables that are difficult to control.

At the same time, the relative performance and influencing factors of the battery system are coupled with each other, and the electrochemical process, which is affected usually by the battery structure, the battery temperature field, so the traditional experimental research methods is difficult to achieve a comprehensive and accurate study.

With the rapid development of computer technology, numerical simulation method has become an important way to analyze and solve complex problems, design and optimization. In the field of lithium-ion batteries, through the computer numerical simulation technology, the establishment of mathematical physics and electrochemical model can fully and systematically capture the battery working process of the interaction mechanism and optimize the battery system design.

There are some challenges in battery model and numerical simulation method, which including the complexity of battery electrochemical reaction, contradictions between system simulation speed and simulation accuracy, variability of battery performance within different situation, variability and complexity of battery operating environment and battery aging and life.

The simulation should be involved consider in that precision and real-time; equivalent circuit model taking into account the simulation accuracy and speed; operating conditions; full charge release cycle; dynamic charging/discharging cycles; operating temperature; charging/discharging current range, and so on.

\section{The principle and charging/discharging characteristics of lithium-ion battery}

Lithium-ion battery is mainly composed of two electrodes, electrolytes and porous partitions as stated in [6]. The active material lithium ion is provided by a lithiumcontaining transition metal oxide such as $\mathrm{LiCoO} 2, \mathrm{LiNiO} 2, \mathrm{LiMn} 2 \mathrm{O} 4$ in positive electrode. The working material for lithium ions embedding in is coke, graphite, amorphous carbon and so on in negative electrode. Electrolyte is dissolved in the lithium salt of organic solvents, lithium salts are LiPF6, LiAsF6, LiClO4, etc., and organic solvents are mainly ethylene carbonate, propylene carbonate, dimethyl carbonate and chlorocarbonate. The diaphragm is porous, electrically insulated, and is physical barriers between the poles that allow ions to pass through. The separator 
material of lithium-ion batteries used in are polyethylene or polypropylene. Lithiumion batteries comply with the "rocking chair" principle in the charging and discharging process. When charging, $\mathrm{Li}+$ leaving the positive layer of the layered structure, under the action of external voltage, through the electrolyte, porous separator, migrate to the underlying structure of the negative, and the electrons flow from the positive to the negative through external wire. At the time of discharging, $\mathrm{Li}+$ leaves the negative layer of the lamellar structure, through the porous separator, migrating to the positive structure of the layered structure, and electrons flow in external circuit to form the current, so the chemical energy conversion is achieved.

The redox reaction occurring at the positive and negative electrodes is:

Positive reaction:

$$
\begin{gathered}
\mathrm{Li}_{1-x} \mathrm{MO}_{2}+x \mathrm{Li}^{+}+x e^{-} \stackrel{\text { discharging }}{\longrightarrow} \mathrm{LiMO}_{2} \\
\mathrm{LiMO}_{2} \stackrel{\text { charging }}{\longrightarrow} \mathrm{Li}_{1-x} \mathrm{MO}_{2}+x \mathrm{Li}^{+}+x e^{-}
\end{gathered}
$$

Negative reaction:

$$
\begin{gathered}
\mathrm{Li}_{x} \mathrm{C}_{6} \stackrel{\text { discharging }}{\longrightarrow} x \mathrm{Li}^{+}+x e^{-}+C_{6} \\
\mathrm{Li}^{+}+x \mathrm{e}^{-}+\mathrm{C}_{6} \stackrel{\text { charging }}{\longrightarrow} x \mathrm{Li}_{x} C_{6}
\end{gathered}
$$

\section{The equivalent circuit model of Lithium-ion battery and research method}

\subsection{The equivalent circuit model of Lithium-ion battery}

An effective battery model is indispensable in designing important systems such as electric vehicles. Sub-items of the battery model should include battery charging/discharging characteristics, state of charge (SOC) and state of health (SOH) estimation, algorithm development, system optimization, and real-time simulation of battery management system design. Among them, the important sub-item is the battery charging/discharging characteristics, SOC and $\mathrm{SOH}$ estimation as stated in $[7,8]$.

Here the Thevenin equivalent circuit model is used. Thevenin equivalent circuit model is a battery equivalent circuit model based on resistive and capacitive properties of batteries and Thevenin theorem. Resistive and capacitive characteristics of the battery mainly capacitive impedance raised from polarization phenomena and ohmic resistance of the battery as stated in $[9,10]$. The capacitive impedance produced from polarization phenomena can be simulated with the resistor $\mathrm{R}$ and the capacitor $\mathrm{C}$, whereby shown in Figure 1. Where R0 is the internal ohmic resistance of the battery, $\mathrm{R} 1$ and $\mathrm{C} 1$ are the polarization resistance and the polarization capacitance, both of which form the RC circuit to describe the polarization of the battery. 


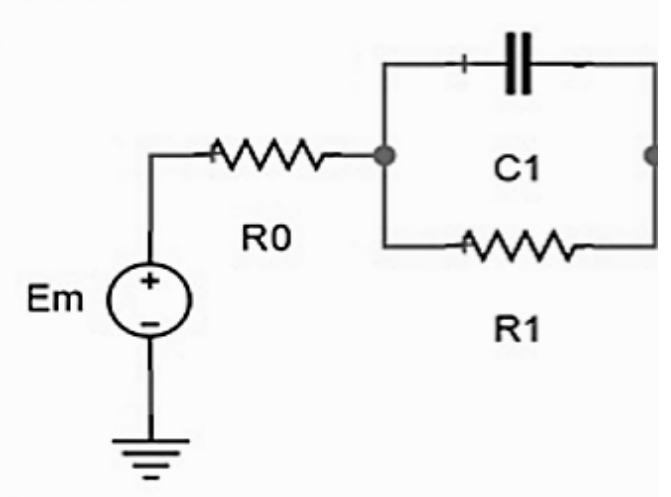

Fig. 1. The Thevenin equivalent circuit model of Li-ion battery

In the Thevenin equivalent circuit model, the following equations can be obtained:

$$
\begin{gathered}
E_{m}=R_{0} i_{t}+R_{1} i_{r t} \\
i_{t}=i_{c t}+i_{r t} \\
i_{c t} / C_{1}=R_{1} \frac{d i_{r t}}{d t}
\end{gathered}
$$

Where $E_{m}$ is the open circuit voltage of battery, $i_{t}$ is the operating current (when charging it is positive, and when discharging it is negative). The Thevenin model has relatively simple structure, the elements have clear physical meaning, and can accurately describe the working characteristics of the battery, but the shortcomings of the model is the open circuit voltage $\mathrm{E}$ as a fixed value, and in the actual work process, the open circuit voltage E of the battery will change. So the Thevenin model should be extended with several new capacitors and resistors, and the $2 \mathrm{RC}$ equivalent circuit model of Li-ion battery is established and shown in Fig. 2.

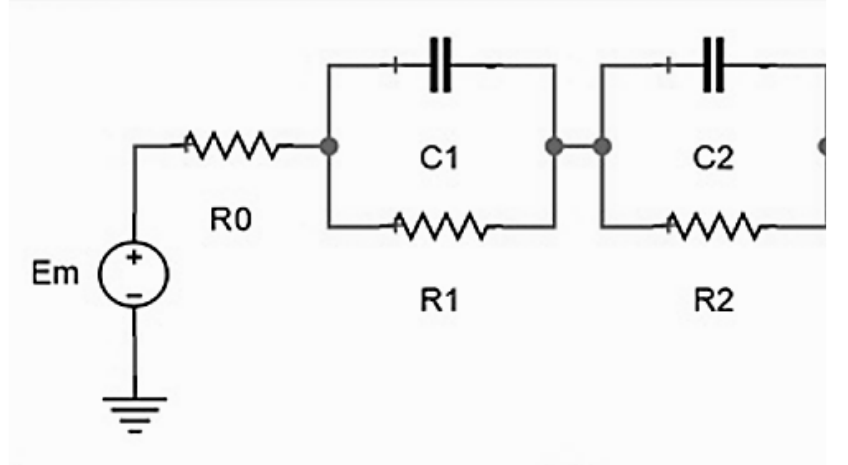

Fig. 2. The $2 \mathrm{RC}$ equivalent circuit model with two time constant, internal resistance and open circuit voltage 
For system-level development and control applications, because of its relatively simplicity, it is preferred use of the equivalent circuit model on battery research. Engineering uses the equivalent circuit model to model the thermal-electrical characteristics of battery, with parametric techniques that combine the model and experimental measurements to optimize the nonlinear elements of the equivalent circuit.

In this paper, the first-order and two-order RC equivalent circuit model is used as the circuit model of lithium ion battery with simplicity and good real-time performance. The battery charge-discharge behavior and thermal effect are simulated and analyzed, and the experimental results are optimized based on the model.

\subsection{The research method}

The first step in developing an accurate battery model is to build and parameterize the equivalent circuit to reflect the nonlinear behavior of the battery and the dependency on temperature, SOC, SOH and current. This dependency is unique to the electro-chemistry character of each cell and needs to be determined based on the measurements on the battery cell.

$\mathrm{SoC}$ is defined as the ratio of the remaining capacity of the battery to the rated capacity under the same conditions at a certain discharge rate as stated in $[11,12]$. The formula is as follows:

$$
\begin{gathered}
\text { SoC }=\frac{Q_{c}}{Q_{N}}=\left[\mathrm{Q}_{N}-\mathrm{Q}\left(\mathrm{I}_{n}\right)\right] / \mathrm{Q}_{N} \\
\mathrm{Q}\left(\mathrm{I}_{n}\right)=\int_{t 0}^{t} \mathrm{I}_{n} d t
\end{gathered}
$$

Where: $Q_{c}$ is the battery remaining capacity, $Q_{N}$ is the battery rated capacity, $\mathrm{Q}\left(\mathrm{I}_{n}\right)$ for the standard discharge current, and $\mathrm{I}_{n}$ is t0-t time the battery release power.

SoC has four aspects of the significance: (1) to maintain the uniformity of battery performance, and ultimately to extend the battery charge and discharge cycle life purposes. (2) To avoid the battery over discharge, overcharge, to protect the battery and vehicle safety. (3) Rational allocation of energy, more effective use of limited energy. (4) Forecast the remaining mileage of the vehicle.

Power battery SoC estimation problem is a typical non-linear, high precision and complex scientific and technical problems, real-time accurate estimation is very difficult. Many factors affect the battery SoC, summed up mainly include: charge and discharge rate, charge and discharge times, polarization effect, temperature, selfdischarge, battery aging. At present, the common research on SoC estimation method of power battery mainly includes: discharge experiment method, open circuit voltage method, Anshi measurement method, artificial neural network method, Kalman filter method and so on.

An-hour measurement method is the most commonly used method for SoC estimation. It calculates the amount of electricity delivered from the battery or the battery power as a simple hour. If the charge and discharge start state is SoC0, then the current state of the $\mathrm{SoC}$ is: 


$$
\mathrm{SoC}=\mathrm{SoC}_{0}-\frac{1}{\mathrm{Q}_{N}} \int_{t 0}^{t} \eta I d t
$$

Where $Q_{N}$ is the rated capacity; I is the battery current; $\eta$ is the charge-discharge efficiency.

The core idea of Kalman filter theory is to use the minimum mean square error as the optimal estimation criterion to find a set of recursive estimation algorithm, which is based on the state space model of signal and noise, using the estimated value of the previous time and Time estimates of the state variables to obtain estimates of the current time. Applying to battery SoC estimates that the battery is seen as a power system, $\mathrm{SoC}$ is an internal state of the system. The core of the $\mathrm{SoC}$ algorithm is a set of recursive equations that include $\mathrm{SoC}$ estimates and reflect the estimation error and covariance matrix. The covariance matrix is used to give the estimated error range. This method is suitable for the estimation of linear systems in white noise environment with high accuracy and real-time performance.

The discrete system of nonlinear systems can be expressed as:

$$
\begin{aligned}
\mathrm{X}(\mathrm{k}+\mathrm{1}) & =\mathrm{f}[\mathrm{k}, \mathrm{X}(\mathrm{k})]+\mathrm{G}(\mathrm{k}) \mathrm{W}(\mathrm{k}) \\
\mathrm{Z}(\mathrm{k}) & =\mathrm{h}[\mathrm{k}, \mathrm{X}(\mathrm{k})]+\mathrm{V}(\mathrm{k})
\end{aligned}
$$

It is assumed here that there is no control input, and the process noise is assumed to be a Gaussian white noise with a mean value of zero, and the noise distribution trix $G(k)$ is known. Among them, the observed noise $V(k)$ is also a Gaussian white noise with additive mean zero. It is assumed that the process noise and the observed noise sequences are independent of each other and have an initial state estimate $\widehat{\mathrm{X}}(0 \mid 0)$ and a covariance matrix $P(0 \mid 0)$. The extended Kalman filter algorithm is as follows:

$$
\begin{gathered}
\widehat{\mathrm{X}}(\mathrm{k} \mid k+1)=\mathrm{f}(\widehat{\mathrm{X}}(\mathrm{k} \mid k)) \\
P(\mathrm{k}+1 \mid k)=\Phi(\mathrm{k}+1 \mid k) \mathrm{P}(\mathrm{k} \mid k) \Phi^{*}(\mathrm{k}+1 \mid k)+\mathrm{Q}(\mathrm{k}+1) \\
\widehat{\mathrm{X}}(\mathrm{k}+1 \mid k+1)=\widehat{\mathrm{X}}(\mathrm{k}+1 \mid k)+\mathrm{K}(\mathrm{k}+1)[\mathrm{Z}(\mathrm{K}+1)-\mathrm{h}(\widehat{\mathrm{X}}(\mathrm{k}+1 \mid k))] \\
P(k+1)=[\mathrm{I}-\mathrm{K}(\mathrm{k}+1) \mathrm{H}(\mathrm{k}+1) P(\mathrm{k}+1 \mid k)]
\end{gathered}
$$

Where the state transition $\Phi(\mathrm{k}+1 \mid k)$ and the measurement matrix $\mathrm{H}(\mathrm{k}+1)$ are represented by the Jacobian matrix of the sum.

\section{The Result of Simulation of Model and Discussion}

The charging current using steady-state charging to $30 \mathrm{~A}$ current charging 0.6 hours, the charging voltage of $4.2 \mathrm{~V}$. The discharge using the interval of dynamic discharge, each discharge period of $30 \mathrm{~A}$ current, discharge time of 0.05 hours, as shown in Fig. 3. 
When charging, the battery open voltage curve found that the battery voltage from $3.7 \mathrm{~V}$ stable linear growth to $4.2 \mathrm{~V}$. When discharging, with the time of discharging and discharging depth increases, the battery voltage gradually reduced from $4.2 \mathrm{~V}$ to $4.07 \mathrm{~V}, 3.93 \mathrm{~V}, 3.79 \mathrm{~V}$, and so on. The battery voltage is reduced once, as shown in Fig. 4.

At the same time, the battery temperature changes can be observed by the battery temperature - working state curve, charging, with the charging time to increase the temperature, up to $34 \square$, charging stopped, the temperature rise trend, the temperature began to decline. When the discharge starts, the battery starts to warm up. At the end of each discharge period, the battery temperature begins to drop. As shown in Fig. 5.

SoC state curve also accurately reflects the charge and discharge process on the impact of SoC. Charging process, SoC linear increase. During the discharge process, SoC is gradually reduced in each discharge period. As shown in Fig. 6.

At the same time, some of the details of the charging and discharging process reflecting the battery charging and discharging transient characteristics.

When the charge is stopped and the discharge is stopped, the battery open circuit voltage always has a transition mutation: if the charge is stopped, the voltage has a reddish process and then recovery to level. When the discharge period is halted, the battery open circuit voltage has a downhill and rapid rebound and then resume a smooth process.

The temperature change is also mutated, when battery discharging, from the discharging gap when the temperature is slow down and changes to rapidly rise. When the discharge period is suspended, the temperature declines slowly, and thus cyclical changes.

The simulation results reflects that the charging and discharging of the li-ion battery has complex characteristics, and the model of battery can provide accurate results for battery SOC estimation.

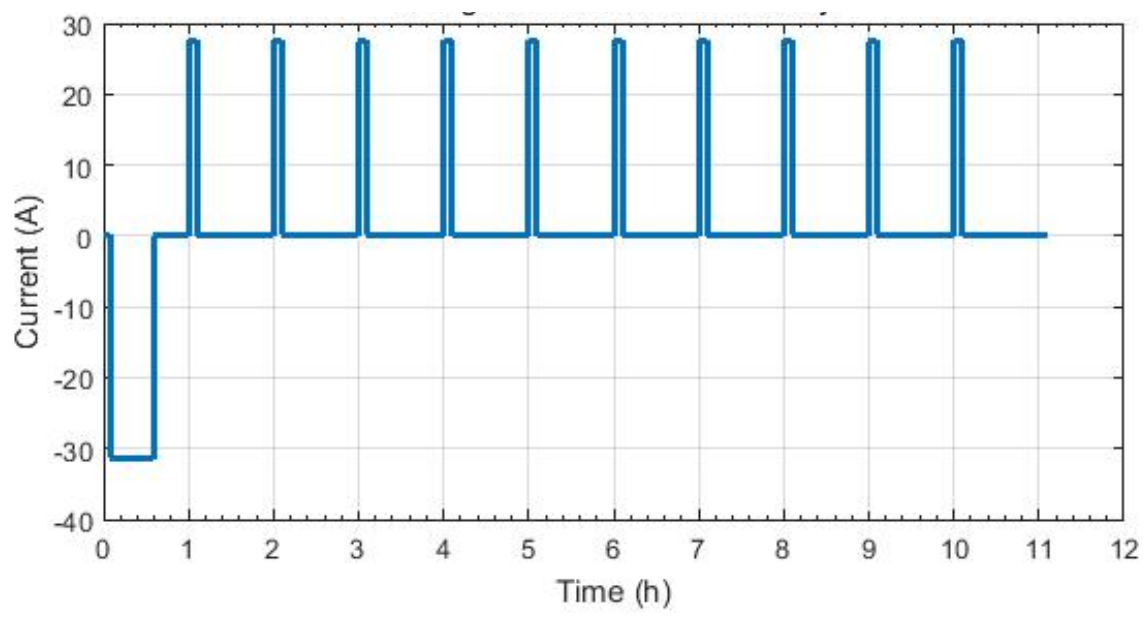

Fig. 3. The Current - Time Curve of Lithium-ion Battery in discharging 


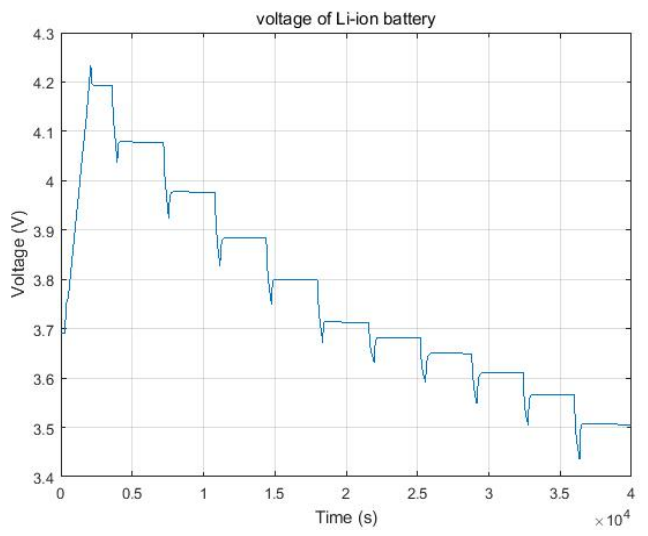

Fig. 4. The open voltage curve of Lithium-ion battery in discharging

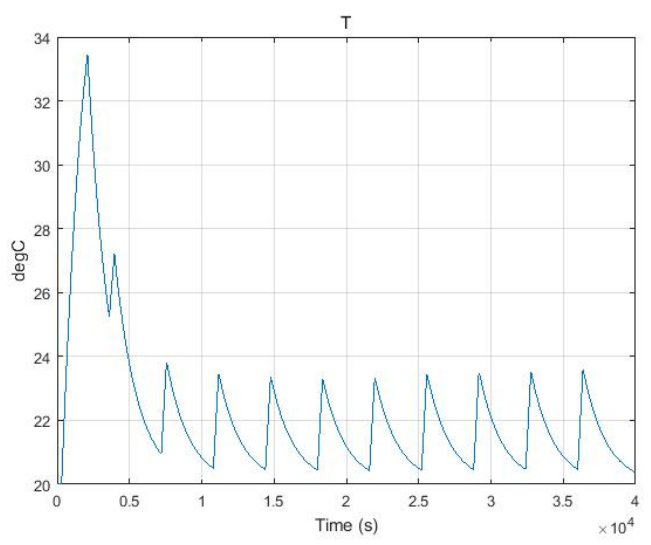

Fig. 5. The temperature curve of Lithium-ion battery in discharging

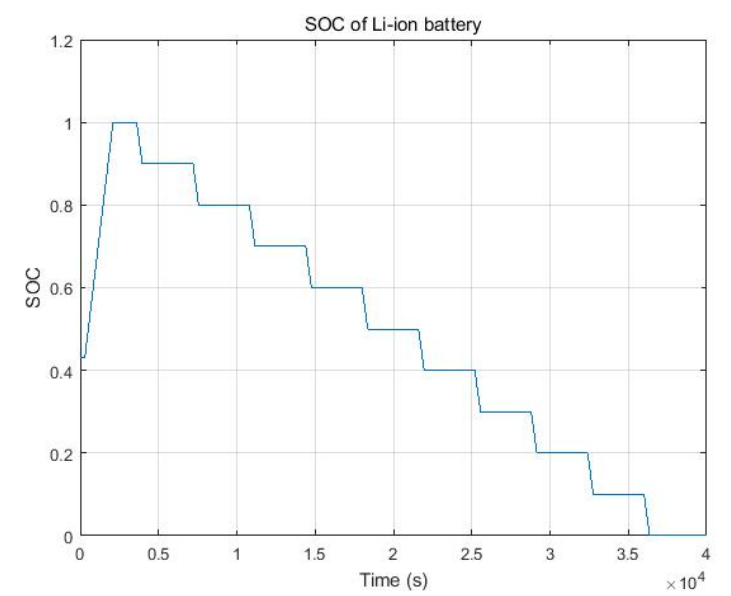

Fig. 6. The SOC curve of lithium-ion battery in discharging 


\section{Conclusion}

The circuit model of lithium ion battery was established by using the second - order RC circuit model, and the battery was simulated dynamically. Through the parameter setting, this model can carry on the simulation research in the complex situation. The current, open circuit voltage, SOC state and battery temperature change of the battery under different charge and discharge are studied. This simulation model is helpful for the optimization and design of lithium ion batteries. It is as the research objects that Lithium-ion batteries with positive and negative materials of LiyMn2O4 and LixC6 were selected.

A mathematical model is made for simulating the electrochemical behavior of Lithium-ion batteries. It is established that the electrochemical cell model and the one $\mathrm{RC}$ cell model based on aforementioned. The case of voltage, current, temperature, SOC and the charging/discharging characteristics were studied. The model is dynamic, and it reflects the transient state of the battery output. The results show that accurate battery charging/discharging strategy management and SOC measurement can be achieved.

\section{Acknowledgment}

Author's research project has accepted funding from the Nature Science Fund of Shandong Province (the program NO. is ZR2015EL041) and Heze University Nature Science Fund (the program NO. is XY14KJ06).

\section{$7 \quad$ References}

[1] Sabine Piller, Marion Perrin, Andreas Jossen. Methods for State Of charge Determination and Their Applications. Journal of Power Sources. 2001.96(1).113 120.

[2] S.Malkhandi. Fuzzy logic-based learning system and estimation of state of charge of leadacid battery. Engineering Applications of Artificial Intelligence. 2006.19(5).479 485.

[3] S. Grewal, D. A. Grant. A novel technique for modeling the state of charge of lithium ion batteries using artificial neural networks. InProc. IEEEITEC. Bristol Univ. UK. 2001.174 179.

[4] G. L. Plett. Extended Kalman filtering for battery management systems of LiPB-based HEV battery packs. Part 3. State and parameter estimation. Journal of Power Sources. 2004. 134(2). 277 292. https://doi.org/10.1016/j.jpowsour.2004.02.033

[5] A. P. Sage, G. W. Husa. Adaptive Filtering with Unknown Prior Statistics. Proceedings of Joint Automatic Control Conference.1969. 760 769.

[6] K.K.C. Yu, N.R. Watson, J. Arrillaga. An adaptive Kalman filter for dynamic harmonic state estimation and harmonic injection tracking. IEEE Trans. Power Delivery. 2005.20(2).1577 1584.

[7] L. Jetto, S. Longhi, G. Venturini. Development and experimental validation of anadaptive extended Kalman filter for localization of mobile robots. IEEE Robotics and Automation, 1999, 15(2). 219 229. https://doi.org/10.1109/70.760343 
[8] M. W. Verbrugge, R. S. Conell. Electrochemical and thermal characterization of battery modules commensurate with electric vehicle integration. Journal of the Electrochemical Society, 2002, 149(1). A45 A53. https://doi.org/10.1149/1.1426395

[9] Fei Zhang, Guangjun Liu, Member, Lijin Fang. A Battery State of Charge Estimation Method with Extended Kalman Filter. Proceedings of the 2008 IEEE/ASME, Xi'an, China, July 2 5, 2008. 1008 1013.

[10] B. S. Bhangu, P. Bentley, D. A. Stone, C. M. Bingham. Observer techniques for estimating the State-of-Charge and State-of-Health of VRLABs for Hybrid Electric Vehicles. IEEE Conf. Vehicle Power and Propulsion. 2005. 780 789. https://doi.org/10.1109/vppc. 2005.1554646

[11] Lars Johannesson, Stefan Pettersson, Bo Egardt. Predictive energy management of a 4QT series-parallel hybrid electric bus. Control Engineering Practice. 2009 (12). 886 896. https://doi.org/10.1016/j.conengprac.2009.07.004

[12] Matthieu Dubarry, Bor Yann Liaw, Mao-Sung Chen, Sain-Syan Chyan, Kuo-Chang Han, Wun-Tong Sie, She-Huang Wu. Identifying battery aging mechanisms in large format Li ion cells. Journal of Power Sources. 2010 (7). 656 673.

\section{Author}

Zhai Haizhou is associated professor of Heze University, Heze City, Shandong Province, PR China. His works focus on electrode materials and control system of liion batteries.

Article submitted 05 March 2017. Published as resubmitted by the author 17 April 2017. 